\title{
COMMENTARY
}

\section{The dual role of mesenchymal stem cells in tumor progression}

\author{
Célia Maria Freitas Gomes \\ See related research by Keramidas et al., http://stemcellres.com/content/4/2/41
}

\begin{abstract}
Mesenchymal stem cells (MSCs) have attracted increasing interest in the field of oncology because of their inherent capacity to migrate and home tumor tissues. The remarkable tropism of MSCs for tumor microenvironments has been exploited in order to use these cells as cellular vehicles to deliver gene therapies or anticancer agents. At functional levels, these cells display chemotactic properties similar to those of immune cells in response to tissue insult and inflammation and secrete a broad range of bioactive biomolecules with an impact on tumor development and a progression through direct actions on tumor cells and the stromal microenvironment. However, the exact contribution of such interactions in tumor progression has not yet been fully clarified, and some concerns remain regarding whether MSCs exert a tumorsuppressive effect or, on the contrary, favor tumor growth. The article by Keramidas and colleagues in this issue of Stem Cell Research \& Therapy provides an interesting contribution to this hot topic.
\end{abstract}

Mesenchymal stem cells (MSCs), also referred to as stromal cells, are non-hematopoietic precursor cells that reside in the bone marrow but can be found in almost all tissues near microvascular niches to maintain homeostasis. These cells display chemotactic properties in response to the release of damage signals, and are mobilized and recruited to injured tissues to participate actively in repair and tissue regeneration. The intrinsic tropism of MSCs for sites of tissue injury is also observed toward tumor lesions because of the production of inflammatory cytokines, chemokines, and growth factors that recruit MSCs to the

\footnotetext{
Correspondence: cgomes@fmed.uc.pt

Laboratory of Pharmacology and Experimental Therapeutics, IBILI - Faculty of Medicine, University of Coimbra, Portugal, Azinhaga de Sta Comba - Celas, 3000-548, Coimbra, Portugal
}

tumor microenvironment. The propensity of MSCs to home to injured tissues and tumor lesions makes them attractive candidates for use in human therapy as cellular vehicles for the delivery of therapeutic agents and the transfer of genetic material. Nevertheless, despite intensive research over the last decade, there is a growing concern about the clinical use of MSC-based therapies in the treatment of cancer. This is because of unanswered questions about homing and engraftment and the controversy regarding whether MSCs induce a tumor-suppressive effect or, in contrast, promote tumor growth and metastasis. The article by Keramidas and colleagues [1] in this issue of Stem Cell Research E Therapy provides new insights into the differential effects of MSCs on tumor growth and angiogenesis. The authors used pre-established models of subcutaneous adenocarcinoma and of lung metastasis in athymic mice. They observed that both systemic or peritumoral administration decreased the growth rate of subcutaneous tumors and led to a delay in disease progression by creating an inhospitable microenvironment for the proliferation of tumor cells. Conversely, they observed that MSCs modified the tumor vasculature, leading to a more structured vascular architecture without altering the overall tumor angiogenesis [1]. The results are in accordance with those of previous studies demonstrating a significant delay in primary tumor growth, lowest tumor incidence, and extended survival in multiple cancer models [2-4]. MSCs secrete a variety of cytokines, chemokines, and growth factors that have an impact on tumor development through a paracrine- or autocrinemediated pathway and have a direct effect on tumor cells or an indirect effect by modifying the surrounding microenvironment which is an integrated and essential part of the tumor tissue. Keramidas and colleagues suggest that the suppressive effect is related to MSC-secreted soluble factors that reduce the proliferative capacity of tumor cells. Some in vitro studies using co-culture systems and MSC-conditioned media have found that MSCs secrete 
soluble factors that alter cell cycle progression, inducing $\mathrm{G}_{1}$-phase cell cycle arrest through downregulation of cell cycle regulators such as cyclin D2 [4]. Some secreted cytokines and chemokines, like CXCL10, interferon-gamma (IFNY), interleukin 6 (IL6), IL8, IFN1 $\beta$, and tumor necrosis factor-alpha, appear to play a role in the inhibitory effect of MSCs on tumor cells [5]. Another MSC-secreted soluble factor with tumor-suppressive effects is the Dickkopf-related protein-1, an inhibitor of the Wnt signaling pathway, which in turn reduced proliferation and induced apoptosis because of downregulation of survival factors [6]. Kucerova and colleagues [7] conducted an interesting study in which they evaluated the effects of adipose tissue-derived MSCs in two different tumor cell lines of melanoma and glioblastoma both in vitro and in vivo. Although the two cell types were exposed to similar MSC-producing pro-inflammatory and pro-angiogenic factors, a pro-survival action on melanoma cells and a tumor-suppressive effect in glioblastoma were observed, suggesting that the overall outcome is greatly dependent on differential responsiveness of tumor cells to paracrine factors secreted by MSCs and on the dynamic and reciprocal interactions between malignant and stromal cells.

One of the tumor-promoting effects of MSCs is attributed to their ability to induce angiogenesis, which is a major factor in the development of tumors. The proangiogenic effect can be mediated indirectly by secretion of pro-angiogenic factors by tumor cells such as vascular endothelial growth factor, stromal-derived factor-1, and platelet-derived growth factor that recruit existing endothelial cells or endothelial progenitor cells (EPCs) or directly through differentiation of MSCs into pericytes, stromal fibroblasts, or endothelial-like cells, promoting the formation of blood vessels [8,9]. Under certain circumstances, MSCs appear to elicit an antiangiogenic response that can be mediated through paracrine pathways that impair the recruitment of EPCs to tumor vasculature or via downregulation of pro-angiogenic factors, ultimately resulting in the abrogation of tumor growth [10]. Keramidas and colleagues [1] show that MSCs induced a remodeling in the tumor vascular architecture without altering the tumor blood volume, suggesting that the tumor growth-inhibitory effects of MSCs they observed in the mouse models were not related to changes in blood supply. The establishment of mature and functional blood vessels in tumors mediated by MSCs, as suggested by the authors, can be seen as a possible approach to improve the delivery of cancer drugs and their efficacy; on the other hand, we cannot forget that a stable functional vascularization could have a tumor-promoting effect due to an improvement in nutrients and oxygen supply. In fact, most studies observed that the promoting effect of MSCs on tumor growth is related to enhanced angiogenesis, induced by secretion of pro-angiogenic factors.
Curiously, this effect is more evident when MSCs were co-transplanted with tumor cells than when administered to established tumors, suggesting that MSC-inducing angiogenesis creates a suitable microenvironment that favors tumor growth at the earliest stages of development. These findings suggest that the timing of MSC administration could be a determinant factor evoking a tumorsuppressive or tumor-promoting tumor-growth effect. Nevertheless, given the complex cross-talk among MSCs and stroma and tumor cells, no simple factor by itself can dictate the fate of tumor cells exposed to MSCs, and future studies will be needed for a better understanding of MSC biology in the context of tumor microenvironment before these cells can serve as a valuable tool in cancer therapy.

\section{Abbreviations}

EPC: Endothelial progenitor cell; IFN: Interferon; IL: Interleukin; MSC: Mesenchymal stem cell.

\section{Competing interests}

The author declares that she has no competing interests.

\section{Published: 29 April 2013}

\section{References}

1. Keramidas M, De Fraipont F, Karageorgis A, Richard MJ, Coll JL, Rome C: The dual effect of mesenchymal stem cell on tumor growth and tumor angiogenesis. Stem Cell Res Ther 2013, 4:41.

2. Klopp AH, Gupta A, Spaeth E, Andreeff M, Marini F 3rd: Concise review: dissecting a discrepancy in the literature: do mesenchymal stem cells support or suppress tumor growth? Stem Cells 2011, 29:11-19.

3. Khakoo AY, Pati S, Anderson SA, Reid W, Elshal MF, Rovira II, Nguyen AT, Malide D, Combs CA, Hall G, Zhang J, Raffeld M, Rogers TB, StetlerStevenson W, Frank JA, Reitz M, Finkel T: Human mesenchymal stem cells exert potent antitumorigenic effects in a model of Kaposi's sarcoma. $J$ Exp Med 2006, 203:1235-1247.

4. Cousin B, Ravet E, Poglio S, De Toni F, Bertuzzi M, Lulka H, Touil I, André M, Grolleau JL, Péron JM, Chavoin JP, Bourin P, Pénicaud L, Casteilla L, Buscail L, Cordelier $P$ : Adult stromal cells derived from human adipose tissue provoke pancreatic cancer cell death both in vitro and in vivo. PLoS One 2009, 4:e6278.

5. Tomchuck SL, Zwezdaryk KJ, Coffelt SB, Waterman RS, Danka ES, Scandurro $A B:$ Toll-like receptors on human mesenchymal stem cells drive their migration and immunomodulating responses. Stem Cells 2008, 26:99-107.

6. Zhu Y, Sun Z, Han Q, Liao L, Wang J, Bian C, Li J, Yan X, Liu Y, Shao C, Zhao RC: Human mesenchymal stem cells inhibit cancer cell proliferation by secreting DKK-1. Leukemia 2009, 23:925-933.

7. Kucerova L, Matuskova M, Hlubinova K, Altanerova V, Altaner A: Tumor cells behaviour modulation by mesenchymal stromal cells. Mol Cancer 2010, 9:129

8. Suzuki K, Sun R, Origuchi M, Kanehira M, Takahata T, Itoh J, Umezawa A, Kijima H, Fukuda S, Saijo Y: Mesenchymal stromal cells promote tumor growth through the enhancement of neovascularization. Mol Med 2011, 17:579-587.

9. Huang WH, Chang MC, Tsai KS, Hung MC, Chen HL, Hung SC: Mesenchymal stem cells promote growth and angiogenesis of tumors in mice. Oncogene 2012. Oct 22 [Epub ahead of print].

10. Ho IA, Toh HC, Ng WH, Teo YL, Guo CM, Hui KM, Lam PY: Human bone marrow-derived mesenchymal stem cells suppress human glioma growth through inhibition of angiogenesis. Stem Cells 2013, 31:146-155.

doi:10.1186/scrt189

Cite this article as: Gomes: The dual role of mesenchymal stem cells in tumor progression. Stem Cell Research \& Therapy 2013 4:42. 